\title{
Genetic analysis of dyslexia candidate genes in the European cross-linguistic NeuroDys cohort
}

\author{
Jessica Becker ${ }^{1,2,37}$, Darina Czamara ${ }^{3,4,37}$, Tom S Scerri ${ }^{5,6}$, Franck Ramus ${ }^{7}$, Valéria Csépe ${ }^{8}$, Joel B Talcott ${ }^{9}$, \\ John Stein ${ }^{10}$, Andrew Morris ${ }^{5}$, Kerstin U Ludwig ${ }^{1,2}$, Per Hoffmann ${ }^{1,2,11}$, Ferenc Honbolygó ${ }^{8}$, Dénes Tóth $^{8}$, \\ Fabien Fauchereau ${ }^{12,13}$, Caroline Bogliotti ${ }^{7}$, Stéphanie Iannuzzi ${ }^{14,15,16}$, Yves Chaix ${ }^{14,15}$, Sylviane Valdois ${ }^{17,18}$, \\ Catherine Billard ${ }^{16}$, Florence George ${ }^{19}$, Isabelle Soares-Boucaud ${ }^{20,21}$, Christophe-Loïc Gérard ${ }^{22}$, Sanne van der Mark ${ }^{23}$, \\ Enrico Schulz ${ }^{24,25}$, Anniek Vaessen ${ }^{26}$, Urs Maurer ${ }^{23,27,28}$, Kaisa Lohvansuu ${ }^{29}$, Heikki Lyytinen ${ }^{29}$, Marco Zucchelli ${ }^{30}$, \\ Daniel Brandeis ${ }^{23,28,31,32}$, Leo Blomert ${ }^{\dagger}, 26$, Paavo HT Leppänen ${ }^{29}$, Jennifer Bruder ${ }^{33}$, Anthony P Monaco ${ }^{5}$, \\ Bertram Müller-Myhsok ${ }^{3,4}$, Juha Kere ${ }^{30,34}$, Karin Landerl ${ }^{35}$, Markus M Nöthen ${ }^{1,2}$, Gerd Schulte-Körne ${ }^{33}$, \\ Silvia Paracchini ${ }^{5,36,37}$, Myriam Peyrard-Janvid ${ }^{30,37}$ and Johannes Schumacher ${ }^{\star, 1,37}$
}

\begin{abstract}
Dyslexia is one of the most common childhood disorders with a prevalence of around $5-10 \%$ in school-age children. Although an important genetic component is known to have a role in the aetiology of dyslexia, we are far from understanding the molecular mechanisms leading to the disorder. Several candidate genes have been implicated in dyslexia, including DYX1C1, DCDC2, KIAA0319, and the MRPL19/C2ORF3 locus, each with reports of both positive and no replications. We generated a European cross-linguistic sample of school-age children - the NeuroDys cohort - that includes more than 900 individuals with dyslexia, sampled with homogenous inclusion criteria across eight European countries, and a comparable number of controls. Here, we describe association analysis of the dyslexia candidate genes/locus in the NeuroDys cohort. We performed both case-control and quantitative association analyses of single markers and haplotypes previously reported to be dyslexia-associated. Although we observed association signals in samples from single countries, we did not find any marker or haplotype that was significantly associated with either case-control status or quantitative measurements of word-reading or spelling in the meta-analysis of all eight countries combined. Like in other neurocognitive disorders, our findings underline the need for larger sample sizes to validate possibly weak genetic effects.
\end{abstract}

European Journal of Human Genetics (2014) 22, 675-680; doi:10.1038/ejhg.2013.199; published online 11 September 2013

Keywords: dyslexia; word-reading; spelling; association study; candidate genes

\section{INTRODUCTION}

Developmental dyslexia is a specific developmental disorder that affects about $5-10 \%$ of school-age children. ${ }^{1,2}$ It is characterized by a severe reading disorder $(\mathrm{RD})$ and spelling problems, which interfere with academic achievement or activities of daily living that require reading skills. ${ }^{3}$ These difficulties cannot be attributed to unimpaired general intelligence, gross neurological deficits, or uncorrected visual or auditory problems. ${ }^{4,5}$ A multifactorial aetiology is most likely,

${ }^{1}$ Institute of Human Genetics, University of Bonn, Bonn, Germany; ${ }^{2}$ Department of Genomics, Life \& Brain Center, University of Bonn, Bonn, Germany; ${ }^{3}$ Max Planck Institute of Psychiatry, Munich, Germany; ${ }^{4}$ Munich Cluster for Systems Neurology (SyNergy), Munich, Germany; ${ }^{5}$ Wellcome Trust Centre for Human Genetics, University of Oxford, Oxford, UK; ${ }^{6}$ Walter and Eliza Hall Institute for Medical Research, Melbourne, Victoria, Australia; ${ }^{7}$ Laboratoire de Sciences Cognitives et Psycholinguistique, Ecole Normale Supérieure, CNRS, EHESS, Paris, France; 8Institute of Cognitive Neuroscience and Psychology, Research Centre of Natural Sciences of the Hungarian Academy of Sciences Budapest, Budapest, Hungary; ${ }^{9}$ School of Life and Health Sciences, Aston University, Birmingham, UK; ${ }^{10}$ Department of Physiology, University of Oxford, Oxford, UK; ${ }^{11}$ Division of Medical Genetics, University Hospital and Department of Biomedicine, University of Basel, Basel, Switzerland; ${ }^{12}$ Human Genetics and Cognitive Functions, CNRS URA 2182, Institut Pasteur, Paris, France; ${ }^{13}$ Sorbonne Paris Cité, Université Paris Diderot, Paris, France; ${ }^{14}$ Unité de Neurologie Pédiatrique, Hôpital des Enfants, Toulouse, France; ${ }^{15} \mid n s e r m$ U825, Hôpital Purpan, Toulouse, France; ${ }^{16}$ Centre de Référence sur les Troubles des Apprentissages, Hôpital Bicêtre, Paris, France; ${ }^{17}$ Laboratoire de Psychologie et NeuroCognition UMR 5105 CNRS, Université Pierre Mendès France, Grenoble, France; ${ }^{18}$ Centre référent pour le diagnostic des troubles du langage et des apprentissages, Département de pédiatrie, CHU Nord, Grenoble, France; ${ }^{19}$ Centre de Référence des Troubles d'apprentissage, CHU Timone, Marseille, France; ${ }^{20}$ Centre de Référence pour les Troubles des Apprentissages, Hospices Civils de Lyon, Hôpital E Herriot, Lyon, France; ${ }^{21}$ Centre Hospitalier Le Vinatier, Bron, France; ${ }^{22}$ Service de Psychopathologie de l'enfant et de l'adolescent, Hôpital Robert Debré, APHP, Paris, France; ${ }^{23}$ Department of Child and Adolescent Psychiatry, University of Zurich, Zurich, Switzerland; ${ }^{24 T U M-N e u r o i m a g i n g ~}$ Center, Technische Universität München, Munich, Germany; ${ }^{25}$ Department of Neurology, Technische Universität München, Munich, Germany; ${ }^{26}$ Department of Cognitive Neuroscience, Faculty of Psychology and Neuroscience \& Maastricht Brain Imaging Institute (M-BIC), Maastricht University, Maastricht, The Netherlands; ${ }^{27}$ Department of Psychology, University of Zurich, Zurich, Switzerland; ${ }^{28}$ Neuroscience Center Zurich, University of Zurich and ETH Zurich, Zurich, Switzerland; ${ }^{29}$ Finnish Center of Excellence in Learning and Motivation Research, Department of Psychology, University of Jyväskylä, Jyväskylä, Finland; ${ }^{0}$ Department of Biosciences and Nutrition, Karolinska Institutet, Huddinge, Sweden; ${ }^{31}$ Zurich Center for Integrative Human Physiology (ZIHP), Zurich, Switzerland; ${ }^{32}$ Department of Child and Adolescent Psychiatry and Psychotherapy, Central Institute of Mental Health, Medical Faculty Mannheim/Heidelberg University, Mannheim, Germany; ${ }^{33}$ Department of Child and Adolescent Psychiatry, Psychosomatics and Psychotherapy, Ludwig-Maximilians-University Munich, Munich, Germany; ${ }^{34}$ Molecular Medicine Program, Biomedicum, University of Helsinki and Folkhälsan Institute of Genetics, Helsinki, Finland; ${ }^{35}$ Department of Psychology, University of Graz, Graz, Austria; ${ }^{36}$ School of Medicine, University of St Andrews, St Andrews, UK

37These authors contributed equally to this work.

†'We would like to express our deepest condolences on the loss of our colleague and friend Leo Blomert, who passed away in 2012.

${ }^{*}$ Correspondence: Dr J Schumacher, Institute of Human Genetics, University of Bonn, Sigmund-Freud-Strasse 25, D-53105 Bonn, Germany. Tel: +49 22828751028 ; Fax: +49 228287 51011; E-mail: johannes.schumacher@uni-bonn.de

Received 25 March 2013; revised 2 August 2013; accepted 7 August 2013; published online 11 September 2013 
caused by interactions between genetic and environmental factors. ${ }^{6}$ Studies have repeatedly indicated that first-degree relatives of affected individuals have a $30-50 \%$ risk of developing the disorder. ${ }^{6,7}$

Genetic linkage studies of dyslexia have identified several loci that may contribute to the disorder. ${ }^{8,9}$ In addition, at some of these loci, association studies or translocation breakpoint mapping have led to the identification of genetic variants associated with disease risk. ${ }^{10}$

DYX1C1 (dyslexia susceptibility 1 candidate 1, MIM 608706) on chromosome $15 \mathrm{q} 21.3$ was identified as a candidate gene by breakpoint mapping of a translocation co-segregating with dyslexia in one Finnish family. ${ }^{11}$ Furthermore, two putative functional variants in DYX1C1 were found to be dyslexia-associated in a population sample of Finnish origin. ${ }^{11}$ Other groups also found DYX1C1 associations in their dyslexia sample, ${ }^{12}$ but also reported an opposite allelic trend with their association findings. ${ }^{13,14}$ It has been speculated that this may be because of a different haplotype structure between samples and populations. DYX1C1 has also been associated with reading and spelling ability in a large unselected group of adolescents from Australia. ${ }^{15}$ Furthermore, it has been shown that dyslexia-associated variants within the promoter region of $D Y X 1 C 1^{16}$ influence the binding affinity of transcription factor complexes. ${ }^{17}$

Two genes have been reported to be associated with dyslexia within the linkage region on chromosome 6p22.2: DCDC2 (doublecortin domain-containing protein 2, MIM 605755) ${ }^{18-20}$ and KIAA0319 (MIM 609269). ${ }^{21,22}$ Independent replications have been reported for both genes: DCDC2 $23-27$ and KIAA0319. ${ }^{27-31}$ The role of KIAA0319 in dyslexia was also supported by the identification of a single variant associated with dyslexia and affecting the gene expression of KIAA0319. ${ }^{30,32}$ In addition, two independent studies have identified an interaction between single nucleotide polymorphisms (SNPs) within DCDC2 and KIAA0319. ${ }^{31,33}$ A recent brain imaging study found support for effects on white matter structure in overlapping regions of human brains for the three dyslexia candidate genes DYX1C1, DCDC2, and KIAA0319.34
On chromosome 2p12, a locus close to the genes MRPL19 and C2ORF3 (also named GCFC2) has been shown to be associated with dyslexia in two independent samples of Finnish and German origin. ${ }^{35}$ However, until now these associations have not been replicated in independent dyslexia samples ${ }^{24}$ but the same genetic variants have been found to be associated with measures of general cognitive abilities. ${ }^{36}$

Conducting association studies of cognitive phenotypes is plagued with challenges, such as the variability in both the initial ascertainment and the subsequent phenotypical assessment of the samples. ${ }^{37,38}$ To address this issue, the NeuroDys Consortium embarked in a large sample collection across eight different European countries applying the same inclusion and exclusion criteria for phenotypic characterization $^{39}$ and collected 958 cases and 1150 controls. In the present study, this sample was used to explore the contribution of the dyslexia candidate genes in such a cross-linguistic cohort. On the basis of existing replication studies, we chose 19 SNPs within the dyslexia candidate genes DYX1C1, DCDC2, KIAA0319, and the MRPL19/ C2ORF3 locus (Table 1), and performed case-control and quantitative (ie, word-reading and spelling) association analyses of single markers and haplotypes.

\section{SUBJECTS AND METHODS}

\section{Subjects}

All parents of children participating in this study gave their written informed consent for participation. The same inclusion and exclusion criteria were applied in all partner countries.

\section{Inclusion and exclusion criteria for all participants}

- Age between 8 and 12 years.

- At least $1 \frac{1 / 2}{2}$ years of formal reading instruction.

- An age-appropriate scaled score of at least 7 on WISC Block Design, and of at least 6 on WISC Similarities (standardized tests of non-verbal and verbal intelligence, respectively, with a population mean $=10$ and $\mathrm{SD}=3^{40}$ ).

Table 1 Genotyped SNPs from the four known dyslexia loci. In total, 19 SNPs were analysed: four SNPs within the MRPL19/C2ORF3 locus, three SNPs within DCDC2, seven SNPs within KIAA0319, and five SNPs within DYX1C1

\begin{tabular}{|c|c|c|c|c|c|}
\hline SNP & Chr & Position $^{\mathrm{a}}$ & Gene & Position relative to gene(s) & Identified by \\
\hline rs1000585 & 2 & $75,676,670$ & MRPL19/C2ORF3 & Upstream of MRPL19, downstream of C2ORF3 & Anthoni et al. ${ }^{35}[\mathrm{FI}, \mathrm{D}]$ \\
\hline rs917235 & 2 & $75,679,327$ & MRPL19/C2ORF3 & Upstream of MRPL19, downstream of C2ORF3 & Anthoni et al. ${ }^{35}[\mathrm{FI}, \mathrm{D}]$ \\
\hline rs714939 & 2 & $75,688,615$ & MRPL19/C2ORF3 & Upstream of MRPL19, downstream of C2ORF3 & Anthoni et al. ${ }^{35}[\mathrm{FI}, \mathrm{D}]$ \\
\hline rs6732511 & 2 & $75,693,241$ & MRPL19/C20RF3 & Upstream of MRPL19, downstream of C2ORF3 & Anthoni et al. ${ }^{35}[\mathrm{FI}, \mathrm{D}]$ \\
\hline rs793862 & 6 & $24,315,179$ & $D C D C 2$ & Intronic & Schumacher et al. ${ }^{19}$ [D] \\
\hline rs807701 & 6 & $24,381,770$ & $D C D C 2$ & Intronic & Schumacher et al. ${ }^{19}$ [D] \\
\hline rs807724 & 6 & $24,386,848$ & $D C D C 2$ & Intronic & Meng et al. ${ }^{18}$ [US] \\
\hline rs4504469 & 6 & $24,696,863$ & KIAA0319 & Coding exonic (missense) & Francks et al. ${ }^{21}$ [UK] \\
\hline rs2179515 & 6 & $24,736,182$ & KIAA0319 & Intronic & Harold et al. ${ }^{31}$ [UK] \\
\hline rs761100 & 6 & $24,740,621$ & KIAA0319 & Intronic & Harold et al. ${ }^{31}$ [UK] \\
\hline rs6935076 & 6 & $24,752,301$ & KIAA0319 & Intronic & Cope et al. ${ }^{22}$ [UK] \\
\hline rs3212236 & 6 & $24,756,434$ & KIAA0319 & Promoter & Harold et al. ${ }^{31}$ [UK] \\
\hline rs9461045 & 6 & $24,757,040$ & KIAA0319 & Promoter & Dennis et al. ${ }^{30}$ [UK] \\
\hline rs2143340 & 6 & $24,767,050$ & KIAA0319 & Intronic & Francks et al. ${ }^{21}$ [UK] \\
\hline rs57809907 & 15 & $53,510,174$ & $D Y X 1 C 1$ & Intronic, 3'UTR, coding exonic (nonsense) (depending on the isoform) & Taipale et al. ${ }^{11}[\mathrm{FI}]$ \\
\hline rs600753 & 15 & $53,546,485$ & $D Y X 1 C 1$ & Coding exonic (missense) & Scerri et al. ${ }^{13}$ [UK] \\
\hline rs17819126 & 15 & $53,577,202$ & $D Y X 1 C 1$ & Coding exonic (missense) & Bates et al. ${ }^{15}$ [AUS] \\
\hline rs3743204 & 15 & $53,577,602$ & $D Y X 1 C 1$ & Intronic (boundary) & Wigg et al. ${ }^{14}[\mathrm{CA}]$ \\
\hline rs3743205 & 15 & $53,577,822$ & $D Y X 1 C 1$ & 5'UTR & Taipale et al. ${ }^{11}$ [FI] \\
\hline
\end{tabular}

according to dbSNP build 130 (hg18)

bPublications that first reported positive association to these SNPs are listed, followed by the origin of the studied sample sets in square brackets: $A U S=A u s t r a l i a, ~ D=G e r m a n y ; ~ C A=C a n a d a ;$ $\mathrm{FI}=$ Finland, UK = United Kingdom, US = United States 
- An attention scale score within the 95th percentile of the age-appropriate norm, either from the Child Behavior Check-List ${ }^{41}$ or from the Conners questionnaire $^{42}$ from the parents.

- The following exclusion criteria from the parental questionnaire: hearing loss; uncorrected sight problems; language of the test not spoken by at least one parent since birth; test language not being the child's school language; child missed school for any period of 3 months or more; formal diagnosis of attention deficit hyperactivity disorder; medication for epilepsy or behavioural problems.

\section{Inclusion criterion for the dyslexia cases}

- More than 1.25 SD below grade level on a standardized word-reading test.

\section{Inclusion criterion for the controls}

- Less than 0.85 SD below grade level on a standardized word-reading test.

The NeuroDys cohort is composed of 958 dyslexia cases and 1150 controls from eight different European countries: Austria, France, Germany, The Netherlands, Switzerland, Finland, Hungary, and the United Kingdom (Table 2).

\section{Phenotypes}

Dyslexia. On top of common inclusion and exclusion criteria (see above), children were classified according to word-reading ability; dyslexic (case) if below $-1.25 \mathrm{SD}$ or control if above $-0.85 \mathrm{SD}$.

Word-reading. With the exception of English, word-reading accuracy and word-reading speed were assessed by presenting word lists under a speeded instruction ('Read as quickly as possible without making mistakes'). Both accuracy and speed were recorded, and converted into a composite wordreading fluency measure (number of words correctly read per minute), then into $Z$-scores based on age- or grade-appropriate norms for each language. In English, reading was not timed and therefore this measure reflects word-reading accuracy only.

Spelling. Standardized spelling tests were given by each contributor. All tests required the spelling of single words dictated in sentence frames and the number of spelling errors were counted. Grade-specific $Z$-scores were calculated based on age- or grade-appropriate norms for each language.

\section{Genotyping}

Samples were genotyped for 19 SNPs using the Sequenom MassARRAY system (Sequenom, San Diego, CA, USA) in one of three laboratories. The UK samples were genotyped at the Wellcome Trust Centre for Human Genetics (Oxford, UK), the Finnish samples were genotyped at the Mutation Analysis Facility (MAF) of the Karolinska Institutet (Stockholm, Sweden), whereas the remaining six sample sets (from Austria, France, Germany, Hungary, Switzerland, and The Netherlands) were genotyped at the Life \& Brain Center (Bonn,
Germany). For quality controls we included intra- and inter-plate duplicates and no genotype inconsistencies were observed. Furthermore, we added negative controls $\left(\mathrm{H}_{2} \mathrm{O}\right)$ on each 384-well plate to exclude contamination. SNP clusterplots were visually checked and manually corrected if necessary. For all sample sets independently, SNPs with a minor allele frequency $<1 \%$ and a call rate $<95 \%$ were excluded. All SNPs were in Hardy-Weinberg equilibrium $(P>0.01)$ and individuals with a call rate $<85 \%$ were excluded. After these quality control measures, 15 of the 19 SNPs genotyped remained in common for all the eight sample sets (Supplementary Tables 1 and 2).

\section{Statistical analyses}

Tests for heterogeneity were conducted using Genepop (http://genepop.curtin. edu.au/). Association analyses for single markers as well as for haplotypes were performed using PLINK (http://pngu.mgh.harvard.edu/ purcell/plink/). Z-score-based meta-analysis was calculated in $\mathrm{R}$ (http://www.r-project.org/). Haplotypes were selected based on previously published positive associations, that is, rs917235-rs714939 (G-G), rs1000585-rs917235-rs714939 (G-G-G), and rs917235-rs714939-rs6732511 (G-G-C) for the MRPL19/C2ORF3 locus ${ }^{35}$ and rs793862-rs807701 (A-C) for the DCDC2 locus. ${ }^{19}$

Correction for multiple testing was performed using the Bonferroni method. The correction based on 19 single markers and 4 haplotypes - analysed for three traits (case-control, word-reading, and spelling) - results in a significance threshold of $P=0.00072$ ( $=0.05 / 69$ tests).

\section{RESULTS}

We performed a genetic heterogeneity analysis of all sample sets included in the study to assess whether we could analyse the whole data set as a single sample or as a meta-analysis. For this, we tested at each locus if alleles were drawn from the same distribution in all eight populations. This analysis revealed significant inter-population differences between the eight sample sets but with no significant differences in allele frequencies for the sample sets from Central Europe ('CE' sample, Supplementary Table 3). We therefore performed a casecontrol analysis in each of the eight sample sets separately, followed by a meta-analysis across the 'CE' samples (580 cases and 625 controls from Austria, France, Germany, Switzerland, and The Netherlands) and a meta-analysis across all samples from the NeuroDys cohort ('All' sample: 958 cases and 1150 controls, Table 2).

\section{Case-control association study}

SNPs. In the single marker case-control analysis of each separate sample set, several SNPs reached nominal significance $(P<0.05)$. These included two SNPs from DYX1C1 tested in the Dutch sample and one SNP from DCDC2 tested in the Hungarian sample (Supplementary Table 4). However, none of these SNPs withstood correction for multiple testing. In the meta-analysis of the 'CE' and 'All' samples, no single SNP reached nominal association (Table 3).

Haplotypes. Furthermore, we tested if any previously reported haplotypes showed association using the case-control status. Only the rs793862-rs807701 haplotype from the DCDC2 locus showed nominal

Table 2 Size and composition of the NeuroDys cohort

\begin{tabular}{|c|c|c|c|c|c|c|c|c|c|c|}
\hline & \multicolumn{5}{|c|}{ 'CE' sample } & & & & \multirow[t]{3}{*}{ 'CE' sample } & \multirow{3}{*}{ 'All' sample } \\
\hline & \multicolumn{8}{|c|}{ 'All' sample } & & \\
\hline & Austria & France & Germany & Netherlands & Switzerland & Finland & Hungary & UK & & \\
\hline Cases & 148 & 138 & 157 & 107 & 30 & 175 & 78 & 125 & 580 & 958 \\
\hline Controls & 199 & 68 & 199 & 116 & 43 & 161 & 155 & 209 & 625 & 1150 \\
\hline Total & 347 & 206 & 356 & 223 & 73 & 336 & 233 & 334 & 1205 & 2108 \\
\hline
\end{tabular}


Table 3 Single marker meta-analysis in the 'CE' and 'All' samples. Association results are given for the case-control analysis and for both quantitative measurements, word-reading and spelling

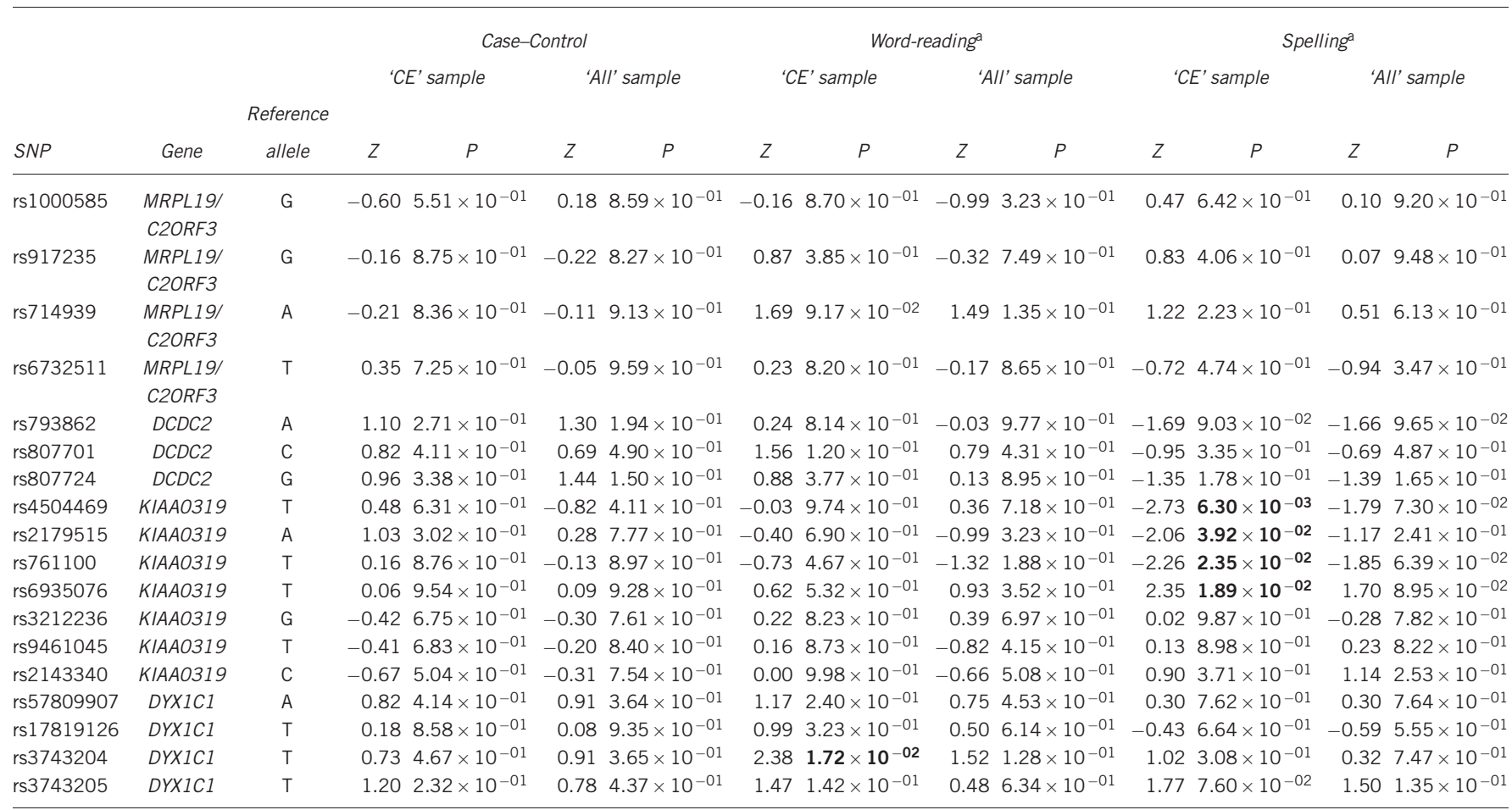

Nominal significant $P$-values $<0.05$ are depicted in bold, Bonferroni-corrected $P$-value is $0.00072(=0.05 / 69)$.

$Z$ defines the test-statistic of the meta-analysis.

anly the dyslexia cases were analysed.

Table 4 Haplotype meta-analysis in the 'CE' and 'All' sample. Association results are given for the case-control analysis and for both quantitative measurements, word-reading and spelling

\begin{tabular}{|c|c|c|c|c|c|c|c|c|c|c|c|c|c|}
\hline \multirow[b]{3}{*}{ SNP combination } & \multirow[b]{3}{*}{ Haplotype } & \multirow[b]{3}{*}{ Gene } & \multicolumn{4}{|c|}{ Case-Control } & \multicolumn{4}{|c|}{ Word-reading ${ }^{a}$} & \multicolumn{3}{|c|}{ Spellinga } \\
\hline & & & \multicolumn{2}{|c|}{ 'CE' sample } & \multicolumn{2}{|r|}{ 'All' sample } & \multicolumn{2}{|c|}{ 'CE' sample } & \multicolumn{2}{|c|}{ 'All' sample } & \multicolumn{2}{|c|}{ 'CE' sample } & 'All' sample \\
\hline & & & $Z$ & $P$ & $Z$ & $P$ & $Z$ & $P$ & $Z$ & $P$ & $Z$ & $P$ & $P$ \\
\hline rs917235-rs714939 & GG & $\begin{array}{l}\text { MRPL19/ } \\
\text { C2ORF3 }\end{array}$ & 0.59 & $5.58 \times 10^{-01}$ & 0.35 & $7.30 \times 10^{-01}$ & -0.88 & $3.79 \times 10^{-01}$ & -0.91 & $3.60 \times 10^{-01}$ & -0.3 & $5.58 \times 10^{-01}$ & $-0.019 .93 \times 10^{-01}$ \\
\hline $\begin{array}{l}\text { rs1000585-rs917235- } \\
\text { rs714939 }\end{array}$ & GGG & $\begin{array}{l}\text { MRPL19/ } \\
\text { C2ORF3 }\end{array}$ & 0.02 & $9.82 \times 10^{-01}$ & 0.01 & $9.92 \times 10^{-01}$ & -1.52 & $1.29 \times 10^{-01}$ & -1.36 & $1.74 \times 10^{-01}$ & -0.12 & $9.82 \times 10^{-01}$ & $0.545 .92 \times 10^{-01}$ \\
\hline $\begin{array}{l}\text { rs917235-rs714939- } \\
\text { rs6732511 }\end{array}$ & GGC & $\begin{array}{l}\text { MRPL19/ } \\
\text { C2ORF3 }\end{array}$ & 0.21 & $8.33 \times 10^{-01}$ & 0.35 & $7.28 \times 10^{-01}$ & -1.22 & $2.24 \times 10^{-01}$ & -0.95 & $3.40 \times 10^{-01}$ & 0.67 & $8.33 \times 10^{-01}$ & $1.082 .80 \times 10^{-01}$ \\
\hline rs793862-rs807701 & $A C$ & $D C D C 2$ & 0.88 & $3.81 \times 10^{-01}$ & 1.24 & $2.15 \times 10^{-01}$ & 0.37 & $7.09 \times 10^{-01}$ & -0.33 & $7.41 \times 10^{-01}$ & -1.24 & $3.81 \times 10^{-01}$ & $-1.082 .79 \times 10^{-01}$ \\
\hline
\end{tabular}

Nominal significant $P$-values $<0.05$ are depicted in bold, Bonferroni-corrected $P$-value is $0.00072(=0.05 / 69)$.

$Z$ defines the test statistic of the meta-analysis.

anly the dyslexia cases were analysed.

association in the Hungarian sample set (Supplementary Table 5). However, this association did not withstand correction for multiple testing. In the 'CE' and 'All' sample, none of the tested haplotypes showed association with dyslexia (Table 4).

\section{Quantitative trait association study}

In a second step, we performed a quantitative trait analysis using two measurements - word-reading and spelling - for all cases of the eight single samples sets separately. Subsequently, we performed a metaanalysis for the quantitative traits across the cases from the ' $\mathrm{CE}$ ' $(N=580)$ and the 'All' $(N=958)$ samples.

SNPs. For some of the genotyped SNPs, we observed nominal associations with word-reading or spelling in single sample sets
(Supplementary Table 6 and Supplementary Table 8). However, only one marker within DYX1C1 - associated with spelling - withstood correction for multiple testing (rs3743205, $P=2.98 \times 10^{-4}$, $P_{\text {corrected }}=0.0206$; Supplementary Table 8 ) in the Switzerland sample set. The meta-analysis across the 'CE' cases resulted in one nominal association between a DYX1C1 SNP and the quantitative trait wordreading (Table 3). For spelling, four markers within KIAA0319 showed nominal association. However, none of these associations withstood correction for multiple testing (Table 3). In the 'All' sample, we did not observe association for the trait word-reading and spelling (Table 3).

Haplotypes. The haplotype association analysis using the quantitative trait word-reading in each sample set separately revealed four nominally significant haplotypes - three of them in the German 
sample and one in the Hungarian sample. However, none of the haplotypes withstood correction for multiple testing (Supplementary Table 7). Furthermore, we observed three nominally significant associations with haplotypes in the spelling analysis: two haplotypes in the German set and the third haplotype in the set from The Netherlands. Again, none of them remained significant after Bonferroni correction (Supplementary Table 9). The haplotype analysis using the quantitative traits revealed no significant association in the 'CE' or 'All' samples (Table 4).

\section{DISCUSSION}

In the present study, we conducted a candidate gene-association analysis in the NeuroDys cohort, which is composed of 958 individuals with dyslexia and 1150 controls from Austria, Finland, France, Germany, Hungary, Switzerland, The Netherlands, and the UK. Participants to the study were recruited using consistent ascertainment criteria across all countries. ${ }^{39}$ To our knowledge, this study represents the first cross-linguistic genetic association analysis in dyslexia. We tested 19 SNPs and 4 haplotypes previously reported to be associated with dyslexia. The markers were located in the dyslexia candidate genes DYX1C1, DCDC2, KIAA0319, and the MRPL19/C2ORF3 locus. Although we observed several nominal associations in samples from individual countries (Supplementary Tables 4-9), none of them were significantly associated with dyslexia or any quantitative phenotypes (ie, word-reading and spelling) in the whole NeuroDys cohort ('All' sample, Tables 3 and 4).

Different reasons may be causing this lack of association. First, the samples included were of different ethnic origin, and different SNPs or haplotypes may contribute to disease or trait risk in divergent populations. This may be particularly true for the Finnish sample, where differences in the genomic architecture compared with other European populations have been previously reported. ${ }^{43,44}$ Even for samples from Central Europe population-specific haplotypes may exist. $^{45,46}$ Second, it is possible that the genetic risk associated with dyslexia is language dependent. However, this hypothesis seems rather unlikely for the samples from Austria, Germany, and Switzerland as these populations are using the same language (ie, German) and we failed to find any association withstanding multiple testing correction restricting our analyses to these samples (data not shown).

Nevertheless, even if the susceptibility to dyslexia is not language dependent, the necessary adaptation of the common ascertainment scheme and of the test battery to each language's properties and to each local environment may have introduced some heterogeneity. In addition, environmental factors - in particular pre-school (nursery/ kindergarten) education and teaching methods applied in schools are different between countries. Third, one limitation of this study is that we have not included measures that cover the whole spectrum of dyslexia-related traits. ${ }^{38,47}$ Previous association studies have reported an association between some of the herein reported genes and phonological processing, orthographic awareness, auditory memory, and rapid naming. ${ }^{38}$ The missing analysis of relevant subtypes, quantitative measures, or the severity of dyslexia could be a further factor for the lack of association in this study.

Furthermore, it is quite possible that the samples used in this study were underpowered to replicate the associations that have been observed previously. It is a known phenomenon that the genetic effect of SNP associations is often overestimated in initial studies (winner's curse). If DYX1C1, DCDC2, KIAA0319, or the MRPL19/ C2ORF3 locus harbour common risk variants contributing to dyslexia, the use of an underpowered case-control sample seems to be the most likely explanation for our replication failure.
Despite all the above-mentioned general causes to our failure in replicating the associations previously reported, gene-specific factors might also be a cause. For example, studies have shown that KIAA0319 appears to be more relevant in controlling general reading ${ }^{27,28}$ abilities and association with this phenotype is more likely to be detected by quantitative trait analysis. However, we failed to detect any association using quantitative trait analysis but it has to be noted that our sample was selected for representing the lower tail of the reading distribution and therefore is not optimal for testing quantitative traits such as general reading skills. Another example concerns DYX1C1, which was originally implicated in the aetiology of dyslexia in a Finnish dyslexia family by breakpoint mapping. It is possible that this gene represents a genuine dyslexia risk gene and that common risk variants in DYX1C1 are contributing to the phenotype, as supported also by associations with reading and spelling in an unselected adolescent cohort from Australia. ${ }^{15}$ However, it might be also possible that high-penetrance mutations in DYX1C1 or in the other dyslexia candidate genes are only present in some familial cases. In this case, a deep-sequencing approach in families with dyslexia would be more appropriate to find an enrichment of such highpenetrance private mutations.

Genome-wide association studies have been successful in mapping risk genes for many complex traits including neuropsychiatric disorders. It has become clear that the success of these studies largely depends on sample sizes, for example a sample size of several thousand individuals seems to be the requirement for achieving significant associations. ${ }^{48,49}$ A genome-wide association study on such a large dyslexia sample would provide an appropriate approach to identify the still unknown dyslexia risk variants. Therefore we conclude that efforts should focus in collecting samples of adequate size by applying similar ascertainment criteria across different countries as we have done with the NeuroDys Consortium.

\section{CONFLICT OF INTEREST}

The authors declare no conflict of interest.

\section{ACKNOWLEDGEMENTS}

We are grateful to all the participants that took part in the study as well as all psychologists who recruited and tested those participants. The NeuroDys Consortium was funded by the EU [Neurodys, 018696]. The work conducted at the WTCHG was supported by Wellcome Trust grants [076566/Z/05/Z] and [075491/Z/04]; the work in Zurich partly by an SNSF grant [32-108130]. We also thank MAF (Mutation Analysis core Facility) at the Karolinska Institute, Novum, Huddinge. The French part of the project was funded by Agence Nationale de la Recherche (ANR-06-NEURO-019-01 GENEDYS) and Ville de Paris. S Paracchini is a Royal Society University Research Fellow. D Czamara was supported by the Deutsche Forschungsgemeinschaft (German Research Foundation) within the framework of the Munich Cluster for Systems Neurology (EXC 1010 SyNergy).

1 Katusic SK, Colligan RC, Barbaresi WJ, Schaid DJ, Jacobsen SJ: Incidence of reading disability in a population-based birth cohort, 1976-1982, Rochester, Minn. Mayo Clin Proc 2001; 76: 1081-1092.

2 Shaywitz SE, Shaywitz BA, Fletcher JM, Escobar MD: Prevalence of reading disability in boys and girls. Results of the Connecticut Longitudinal Study. JAMA 1990; 264: 998-1002.

3 Shaywitz SE, Fletcher JM, Holahan JM et al: Persistence of dyslexia: the Connecticut Longitudinal Study at adolescence. Pediatrics 1999; 104: 1351-1359.

4 ICD-10-GM Version 2008. Internationale statistische Klassifikation der Krankheiten und verwandter Gesundheitsprobleme, 10. Revision - German Modification -; Kapitel V: 192.

5 American Psychiatric Association: Diagnostic and Statistical Manual of Mental Disorders. Washington, DC: American Psychiatric Association, 2000, 4th edn. Text Revision, pp 51-52. 
6 Fisher SE, DeFries JC: Developmental dyslexia: genetic dissection of a complex cognitive trait. Nat Rev Neurosci 2002; 3: 767-780.

7 Barry JG, Yasin I, Bishop DV: Heritable risk factors associated with language impairments. Genes Brain Behav 2007; 6: 66-76.

8 Williams J, O'Donovan MC: The genetics of developmental dyslexia. Eur J Hum Genet 2006; 14: 681-689.

9 Schumacher J, Hoffmann P, Schmal C, Schulte-Körne G, Nöthen MM: Genetics of dyslexia: the evolving landscape. J Med Genet 2007; 44: 289-297.

10 Scerri TS, Schulte-Körne G: Genetics of developmental dyslexia. Eur Child Adolesc Psychiatry 2009; 19: 179-197.

11 Taipale M, Kaminen N, Nopola-Hemmi J et al: A candidate gene for developmental dyslexia encodes a nuclear tetratricopeptide repeat domain protein dynamically regulated in brain. Proc Natl Acad Sci USA 2003; 100: 11553-11558.

12 Marino C, Citterio A, Giorda R et al: Association of short-term memory with a variant within DYX1C1 in developmental dyslexia. Genes Brain Behav 2007; 6 640-646.

13 Scerri TS, Fisher SE, Francks $C$ et al: Putative functional alleles of DYX1C1 are not associated with dyslexia susceptibility in a large sample of sibling pairs from the UK. J Med Genet 2004; 41: 853-857.

14 Wigg KG, Couto JM, Feng $Y$ et al: Support for EKN1 as the susceptibility locus for dyslexia on 15q21. Mol Psychiatry 2004; 9: 1111-1121.

15 Bates TC, Lind PA, Luciano M, Montgomery GW, Martin NG, Wright MJ: Dyslexia and DYX1C1: deficits in reading and spelling associated with a missense mutation. Mol Psychiatry 2009; 15: 1190-1196.

16 Dahdouh F, Anthoni H, Tapia-Paez I et al: Further evidence for DYX1C1 as a susceptibility factor for dyslexia. Psychiatr Genet 2009; 19: 59-63.

17 Tapia-Páez I, Tammimies K, Massinen S, Roy AL, Kere J: The complex of TFII-I, PARP1, and SFPQ proteins regulates the DYX1C1 gene implicated in neuronal migration and dyslexia. FASEB J 2008; 22: 3001-3009.

18 Meng $\mathrm{H}$, Smith SD, Hager $\mathrm{K}$ et al: DCDC2 is associated with reading disability and modulates neuronal development in the brain. Proc Natl Acad Sci USA 2005; 102 17053-17058.

19 Schumacher J, Anthoni H, Dahdouh F et al: Strong genetic evidence of DCDC2 as a susceptibility gene for dyslexia. Am J Hum Genet 2006; 78: 52-62.

20 Deffenbacher KE, Kenyon JB, Hoover DM et al: Refinement of the 6p21.3 quantitative trait locus influencing dyslexia: linkage and association analyses. Hum Genet 2004 115: $128-138$

21 Francks C, Paracchini S, Smith SD et al: A 77-kilobase region of chromosome 6p22.2 is associated with dyslexia in families from the United Kingdom and from the United States. Am J Hum Genet 2004; 75: 1046-1058.

22 Cope N, Harold D, Hill G et al: Strong evidence that KIAA0319 on chromosome 6p is a susceptibility gene for developmental dyslexia. Am J Hum Genet 2005; 76: 581-591.

23 Lind PA, Luciano M, Wright MJ, Montgomery GW, Martin NG, Bates TC: Dyslexia and DCDC2: normal variation in reading and spelling is associated with DCDC2 polymorphisms in an Australian population sample. Eur J Hum Genet 2010; 18 668-673.

24 Newbury DF, Paracchini S, Scerri TS et al: Investigation of dyslexia and SLI risk variants in reading and language-impaired subjects. Behav Genet 2011; 41: 90-104.

25 Ludwig KU, Schumacher J, Schulte-Körne G et al: Investigation of the DCDC2 intron 2 deletion/compound short tandem repeat polymorphism in a large German dyslexia sample. Psychiatr Genet 2008; 18: 310-312.

26 Wilcke A, Weissfuss J, Kirsten H, Wolfram G, Boltze J, Ahnert P: The role of gene DCDC2 in German dyslexics. Ann Dyslexia 2009; 59: 1-11.
27 Scerri TS, Morris AP, Buckingham LL et al: DCDC2, KIAA0319 and CMIP are associated with reading-related traits. Biol Psychiatry 2011; 70: 237-245

28 Paracchini S, Steer CD, Buckingham LL et al: Association of the KIAA0319 dyslexia susceptibility gene with reading skills in the general population. Am J Psychiatry 2008; 165: 1576-1584.

29 Luciano M, Lind PA, Duffy DL et al: A haplotype spanning KIAA0319 and TTRAP is associated with normal variation in reading and spelling ability. Biol Psychiatry 2007 62: 811-817.

30 Dennis MY, Paracchini S, Scerri TS et al: A common variant associated with dyslexia reduces expression of the KIAA0319 gene. PLoS Genet 2009; 5: e1000436.

31 Harold D, Paracchini S, Scerri T et al: Further evidence that the KIAA0319 gene confers susceptibility to developmental dyslexia. Mol Psychiatry 2006; 11: 1061.

32 Paracchini S, Thomas A, Castro S et al: The chromosome 6p22 haplotype associated with dyslexia reduces the expression of KIAA0319, a novel gene involved in neuronal migration. Hum Mol Genet 2006; 15: 1659-1666.

33 Ludwig KU, Roeske D, Schumacher J et al: Investigation of interaction between DCDC2 and KIAA0319 in a large German dyslexia sample. J Neural Transm 2008; 115: $1587-1589$.

34 Darki F, Peyrard-Janvid M, Matsson H, Kere J, Klingberg T: Three dyslexia suscept ibility genes, DYX1C1, DCDC2, and KIAA0319, affect temporo-parietal white matter structure. Biol Psychiatry 2012; 72: 671-676.

35 Anthoni $\mathrm{H}$, Zucchelli M, Matsson $\mathrm{H}$ et al: A locus on 2p12 containing the co-regulated MRPL19 and C2ORF3 genes is associated to dyslexia. Hum Mol Genet 2007; 16 667-677.

36 Scerri TS, Darki F, Newbury DF et al: The dyslexia candidate locus on 2p12 is associated with general cognitive ability and white matter structure. PLoS One 2012; 7: e50321.

37 Paracchini S, Scerri T, Monaco AP: The genetic lexicon of dyslexia. Annu Rev Genomics Hum Genet 2007; 8: 57-79.

38 Skiba T, Landi N, Wagner R, Grigorenko EL: In search of the perfect phenotype: an analysis of linkage and association studies of reading and reading-related processes. Behav Genet 2011; 41: 6-30.

39 Landerl K, Ramus F, Moll K et al: Predictors of developmental dyslexia in European orthographies with varying complexity. J Child Psychol Psychiatry 2013; 54: 686-694.

40 Wechsler D: Wechsler Intelligence Scale for Children, 4th edn, 2003.

41 Achenbach T: Child Behavior Check-List 2001.

42 Conners CK: Rating scales for use in drug studies with children. Psychopharmacol Bull 1973; 9: 24-84

43 Lao O, Lu TT, Nothnagel M et al: Correlation between genetic and geographic structure in Europe. Curr Biol 2008; 18: 1241-1248.

44 Novembre J, Johnson T, Bryc $\mathrm{K}$ et al: Genes mirror geography within Europe. Nature 2008; 456: 98-101.

45 Nelis M, Esko T, Magi R et al: Genetic structure of Europeans: a view from the North-East. PLoS One 2009; 4: e5472.

46 Salmela E, Lappalainen T, Fransson I et al: Genome-wide analysis of single nucleotide polymorphisms uncovers population structure in Northern Europe. PLoS One 2008; 3 . e3519.

47 Schulte-Körne G, Ziegler A, Deimel W et al: Interrelationship and familiality of dyslexia related quantitative measures. Ann Hum Genet 2007; 71: 160-175.

48 Ripke S, Sanders AR, Kendler KS et al: Genome-wide association study identifies five new schizophrenia loci. Nat Genet 2011; 43: 969-976.

49 C4D Genetics Consortium. A genome-wide association study in Europeans and South Asians identifies five new loci for coronary artery disease. Nat Genet 2011; 43: 339-344.

Supplementary Information accompanies this paper on European Journal of Human Genetics website (http://www.nature.com/ejhg) 